\title{
CONSISTENCY \& COMPATIBILITY (TWO SIDES OF THE SAME COIN)
}

\begin{abstract}
In MCDM to have an index of consistency for pair comparison matrices is a very important matter. This index may help to produce better metrics and of course better answers to the problem, which is the final purpose of majority of the MCDM methods.

To illustrate the relevance of this matter, this paper present an example that take in consideration one of the most important consistency index in the literature, the Saaty's consistency index. Working in a way similar to reductio ad absurdum and using the compatibility index as collaborative tool, we will show that Saaty's consistency index is in fact a good quality index.
\end{abstract}

Keywords: Consistency, Compatibility, Saaty’s consistency index, compatibility index G.

\section{1.- Introduction}

Consistency and compatibility can be seen as two sides of the same coin. Consistency is the measure of coherence with yourself (with your own responses). On the other hand, compatibility is the measure of coherence with someone else. Thus, consistency is the "inner" vision for coherence and compatibility the outer vision. (Of course, has no sense to measure coherence with someone else if first you have not been coherent with yourself).

In this way, a created new metric can be consistent inside, but not necessary compatible with another metric from outside.

This condition can be exploded for an interesting application of compatibility. It could be used to measure the compatibility of two slightly different but still consistent metrics, checking the quality of the new metric compared with the old one in terms of its compatibility. If they are compatible, then one can use both, the new or the old consistent metric to measure in approximately equal conditions, and hence the degree of consistency would be enough in both metrics. In this way, we may measure which is the maximum loss of consistency in a metric that can keep a minimum level of acceptable compatibility.

When it is possible to compare a new metric obtained with some method with the expected or actual metric, then the compatibility index $G$ represent a great tool to test and verify the quality of anew created metric.

\section{2.- Literature Review}

The equations for consistency index and compatibility index used in this study are the following:

Consistency:

$\mathrm{RC}==$ ratio of consistency, $\mathrm{IC}=$ index of consistency, $\mathrm{RI}=$ random index

$\mathrm{RC}=\mathrm{IC} / \mathrm{RI}$.

With: IC $=\left(\lambda_{\max }-1\right) /(n-1) \cdot \lambda_{\max }=$ greatest eigenvalue, $n=$ matrix dimension. 
Compatibility:

$\mathrm{G}=$ index of compatibility

$\mathrm{G}=1 / 2 \Sigma_{\mathrm{i}}\left(\mathrm{a}_{\mathrm{i}}+\mathrm{b}_{\mathrm{i}}\right) * \operatorname{Min}(\mathrm{a}, \mathrm{b}) / \operatorname{Max}(\mathrm{a}, \mathrm{b})_{\mathrm{i}}$.

With: $\sum \mathrm{a}_{\mathrm{i}}=\sum \mathrm{b}_{\mathrm{i}}=1, \mathrm{i}=1, \ldots, \mathrm{n}$. (n= dimension of priority vector).

Note: $G$ is the most accurate compatibility index for weighted environments. Also, is the only one which not presents numerical stability problems in distance measurement within weighted environments.

\section{3.- Hypothesis/Objectives:}

For illustrative purpose, suppose for instance we would like to measure the quality of a created new metric in terms of an old well know metric, based on the following example/problem:

The Hypothetical Problem:

As hypothetical problem we are presenting a hypotheticalcriticaboutthe quality of Saaty's consistency index in pairwise comparison matrices.

The hypothetical critic may say: The index of consistency (Saaty's index) is wrong, since it let pass through values (comparisons) that are not acceptable for common sense.

\section{4.- Research Design/Methodology}

Introducing the actual or old well-knew metric:

Suppose three equal bars of same long as shown in figure 1:

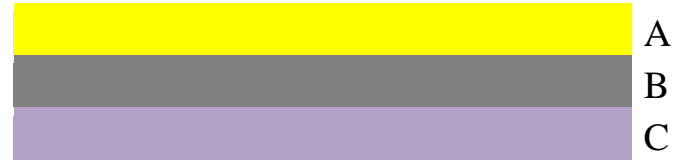

Figure 1: Three bars of equal long

\section{A}

B

Of course, the correct matrix comparison for this situation is the following (consistent) comparison matrix:

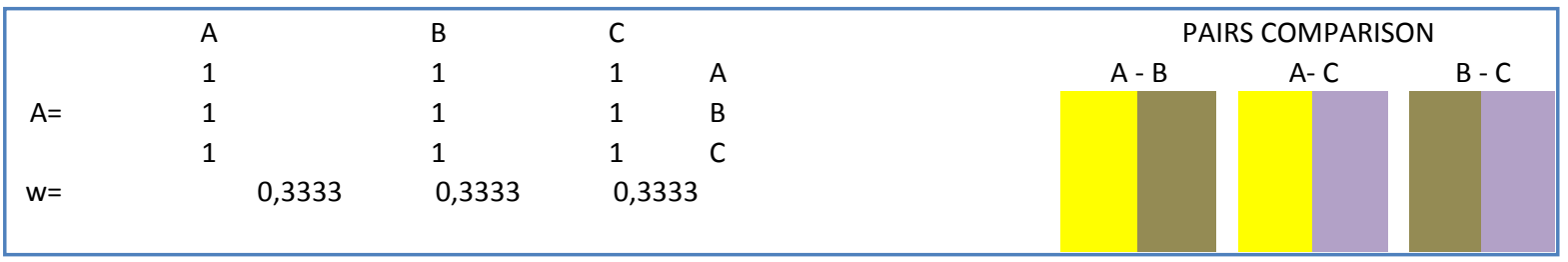

Figure 2a: Pair comparison matrix of three equal bars

The obvious well-knew old metric is given by the priority vector " $w$ " $=1 / 3,1 / 3,1 / 3$, with $100 \%$ of consistency $(\mathrm{RC}=0)$.

Introducing the new metric:

Suppose now that (due to some visualization mistake), the new appreciation about the bars is: 


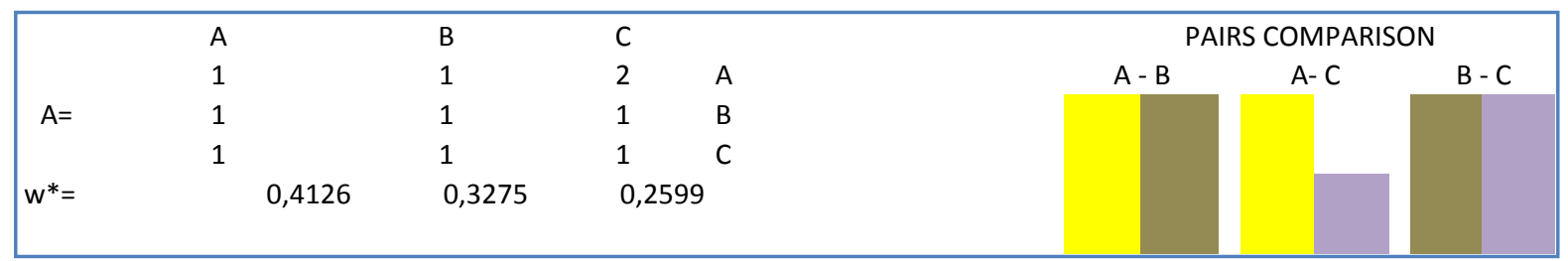

Figure 2b: Pair comparison of three bars changing the value of cell $(1,3)$

The new metric is given by the priority vector " $\mathrm{w}^{*}$ " $=(0.4126,0.3275,0.2599)$, with $\mathrm{RC}=0.05(95 \%$ of consistency), which according to the theory is the maximum acceptable inconsistency for a $3 \times 3$ comparison matrix.

The critic claim that bar comparison A-C has a $100 \%$ of difference (100\% of error), which is not an acceptable error for common sense point of view, (easy to see even at naked eye considering that the three bars are equal).

Also, the global error in the priority vectors is: $15.85 \%$, calculated with the common formula: $\mathrm{e}_{\mathrm{i}}=\operatorname{abs}\left(\mathrm{w}^{*}-\mathrm{w}\right) / \mathrm{w}$, for each coordinate "i" and then take the average over $\mathrm{e}_{\mathrm{i}}$. Thus, we obtain a final error of almost $15.9 \%$.

But, Saaty's consistency index shows $\mathrm{RC}=95 \%$, which is acceptable for a $3 \mathrm{x} 3$ comparison matrix. Hence, the critic claims that Saaty's consistency index is wrong.

If this last sentence is true, then the value of compatibility index of both metrics must be low (that means not sufficient).

By the other side, if this is not true, then we have an absurd result and the sentence must be wrong. (Reductio ad absurdum).

\section{Data/Model Analysis}

Calculating compatibility index $\mathrm{G}$ for both metrics:

Before calculating $\mathrm{G}$, we may visualize two possible misunderstanding for the already described problem:

First: Saaty's consistency index comes from the eigenvalue-eigenvector systemic operator. Hence, this is a systemic approach (does not take care ofsome particular comparison).

Second: The possible error should be measured/qualified by its final result (the resulting metric), not in some prior or middlestep.

The first misunderstanding is explained it by itself.

For the second one, before any calculation, we need to understand what kind of numbers are we dealing with (in what environment we are working), because is not the same to be close to a big priority than to a small one. This is a weighted environment and the measure of the closeness (proximity) has to consider this situation.

We must work in the order topology domain to correctly measure the proximity (closeness) in this environment. To do this correctly two aspects of the information must to be considered: the intensity (the weight or priority) and the degree of coupler between the priority vectors (the degree of projection between the vectors).The index that take good care of this two factors simultaneously is the compatibility index G. 
Summarizing, the two vectors of correct and approximated metric are:

$\begin{array}{llll}\text { Correct metric (priority vector): } & 0.3333 & 0.3333 & 0.3333 \\ \text { Approximated metric (priority vector) } & 0.4126 & 0.3275 & 0.2599\end{array}$

The basic two questions here are:

1. How close is the approximated metric to the correct metric?

2. Is that closeness close enough to be considered compatible?

\section{6.- Limitations}

When evaluating the compatibility index G between the Correct and Approximated vectors, the G value obtained is $=85.72 \%$, which in numerical terms represent almost compatible metrics. $\mathrm{G}=90 \%$ is a threshold to consider two priority vectors as compatible vectors.

Also, $\mathrm{G}=85 \%$ is an acceptable lower limit value for compatibility, especially when not working with physical measures.

\section{7.- Conclusions}

The answers for the two basic questions are:

1. The two metrics are relatively close.

2. They are close enough to be considered compatible (considering that they are not physical measures).

Thus, the final conclusion is that Saaty's consistency index is a good index under this test.

Of course, if anyone still wants to get a better consistency, he/she can do it.

The question is: do we really obtain a better result been totally consistent? And the answer is: probably no,because in real problems we never have the "real" answer (the true or "physical" measurement to contrast).

For instance, in the presented problem one could answer that: $\mathrm{A}-\mathrm{B}=2$; $\mathrm{A}-\mathrm{C}=2$; and $\mathrm{B}-\mathrm{C}=1$; and he/she would be totally consistent, (but consistently wrong).

The new priority vector would be: $\mathrm{w}^{* *}=(0.5,0.25,0.25)$, with $\mathrm{RC}=0$ (totally consistent).

By the other hand, for this case the compatibility index $G$ would be: $71.5 \%$, which means incompatible vectors (two far away metrics). Thus, a totally consistent metric is incompatible with the actual result.

At the end,seems it is better to be approximately correct than consistently wrong.

(The consistency index is just a thermometer not a goal).

The second important conclusion is that compatibility index G can be applied as quality tester for new methods of measurement and new indices,through measuring the degree of similarity of two priority vectors (resultant rankings).

\section{1- Some clarifying notes for final conclusions}

Note1: $\mathrm{RC}=0.05$ is the maximum acceptable value for inconsistency in $3 \mathrm{x} 3$ matrices. Thus, we can not go further with the bar comparison number.That means we cannot put a 3 (or greater) instead of 2 in the cell $(1,3)$. 
Note2: if the metric B is compatible with metric A then we can use metric B as a good approximation of A. This is a useful property when metric A is not available (the most of the times we don't know the correct/exact metric).

Note3:When the same exercise was performed in $4 \times 4$ to $9 x 9$ matrices, putting a(n-1) value in the cell-position (1,n), we obtained similar results for compatibility (sometime even better).

Note4: The threshold value for compatibility index is described in the Garuti's book: "Measuring in weighted environment, moving from metric to order topology" (Knowing when close really means close).

Note5: Next, a graphical difference between two “equal” bars with an error of $15.9 \%$.

Bar approximately $15.9 \%$ larger.

Here, the reader can appreciate how similar (or close) are both bars in global terms. Also, he/she might judge if this difference is admissible or not. Especially consideration has to be made when dealing with intangible criteria (or criteria that have no metric defined).

It is also interesting to note that compatibility index $G$ was $85.7 \%(\geq 85 \%)$, which represent a tolerable compatibility between both metrics old and new.Also, this $85.7 \%$ means $14.3 \%$ of incompatibility (Incompatibility $=1-$ Compatibility). Thus, we may say that incompatibility (or global difference/error) in both metrics is totally acceptable, and this is especially true when dealing with intangible criteria.

Hence, Saaty’s consistency index is adequate for its purpose.

\section{8.- Key References}

1. Garuti, C. 2014. Measuring In Weighted Environments. International Journal of AHP (IJAHP) 2014. (Article).

2. Garuti, C Compatibility of AHP/ANP vectors with known results. Presentation of a suggested new index of compatibility in weighted environments. ISAHP2014 International Symposium of the Analytic Hierarchy Process. 2014.

3. Garuti, C. 2012. Measuring in Weighted Environments: Moving from Metric to Order Topology. Santiago, Chile: Universidad Federico Santa Maria.(Book, 72pp).

4. Garuti, C. A. 2007. Measuring Compatibility in Weighted Environments: When close really means close? International Symposium on AHP, 9, Viña del Mar, Chile. 2007. (Paper)

5. Hilbert, D. 1895 "Ueber die gerade Linie als kürzeste Verbindung zweier Punkte", Mathematische Annalen (Springer Berlin / Heidelberg) 46: 91-96, doi:10.1007/BF02096204, ISSN 0025-5831, IFM 26.0540.02

6. Mahalanobis, P.C. "On the generalised distance in statistics", Proceedings of the National Institute of Science of India 12 (1936) 49-55.

7. Papadopoulos, A.; Troyanov, M. (2014), Handbook of Hilbert Geometry, European Mathematical Society

8. Saaty, L. T. 2010. GROUP DECISION MAKING: Drawing out and Reconciling Differences. Pittsburgh: RWS. (Book, 385pp).

9. Saaty, L.T. 2001. The Analytic Network Process: Decision Making with Dependence and Feedback. (Book, 376pp).

10. Whitaker, R. 2007. Validation Examples of the Analytic Hierarchy Process and Analytic Network Process. Mathematical and Computer Modeling. Vol. 46, pp. 840-859. (Paper). 